\title{
Mineralização óssea em crianças e adolescentes com diabetes melito tipo 1
}

\author{
Bone mineralization in children and adolescents with type 1 diabetes
}

\author{
Deisi M. Vargas ${ }^{1}$, Tatiana Rigotti ${ }^{2}$, Cibelli N.R.M. Gütz ${ }^{2}$, \\ Maria Cláudia S. Lobe ${ }^{3}$, Jucílio de A. Fernades ${ }^{4}$
}

\section{Resumo}

Objetivo: estudar a ocorrência de redução da massa óssea e seus fatores prognósticos em um grupo de pacientes pediátricos com diabetes melito tipo1.

Métodos: estudamos 23 pacientes portadores de diabetes melito tipo 1 , com idade média de 10,9 $\pm 2,9$ anos. Analisou-se a massa óssea, ingestão de cálcio, IMC, peso, estatura, estágio puberal, dose de insulina, duração do diabetes, cálcio, fósforo, fosfatase alcalina, peptídeo $\mathrm{C}$ e hemoglobina glicosilada. A massa óssea foi avaliada em coluna lombar, através de densitometria óssea, e expressa em desvio padrão da média para idade e sexo (DP). A ingestão de cálcio foi calculada através de recordatório alimentar, o IMC, calculado de acordo com a fórmula de Quetelet, e o estágio puberal definido segundo os critérios de Tanner-Whitehouse. Utilizou-se a regressão linear simples para o estudo das relações entre as variáveis e a uMann-Whitney na comparação entre grupos.

Resultados: observamos que a média da densidade mineral óssea (DMO) foi normal (-0,75 $\pm 1,01 \mathrm{DP})$. No entanto, verificamos que $39,1 \%$ dos pacientes apresentavam osteopenia. Ao comparar os dados dos pacientes osteopênicos $(n=9)$ com os não osteopênicos $(\mathrm{n}=14)$, observamos que o peptídeo $\mathrm{C}$ do grupo osteopênico foi superior $(0,56 \pm 0,18 v$ s. $0,29 \pm 0,20, \mathrm{p}<0,05)$. O IMC e o peptídeo C correlacionaram-se com a DMO. A duração do diabetes correlacionou-se negativamente com o peptídeo $\mathrm{C}(\mathrm{p}<0,01)$ e positivamente com a dose de insulina $(\mathrm{p}<0,01)$.

Conclusão: no grupo de diabéticos estudado, encontramos uma freqüência de osteopenia de $39,1 \%$. A presença de osteopenia esteve relacionada a níveis séricos superiores de peptídeo $\mathrm{C}$.

J Pediatr (Rio J) 2003;79(3):253-8: diabetes tipo 1, osteopenia, densidade mineral óssea.

1. Doutora, Dep. de Medicina, Disciplina de Pediatria, Ambulatório de Endocrinologia Pediátrica da Universidade Regional de Blumenau (FURB).

2. Interno do Curso de Medicina da FURB.

3. Mestra, Dep. de Medicina, Disciplina de Pediatria, Ambulatório de Endocrinologia Pediátrica - FURB.

4. Especialista, Centro Catarinense de Densitometria Óssea - Florianópolis, SC.

Fonte financiadora: Programa Institucional de Iniciação Científica PIBIc - CNPq. Universidade Regional de Blumenau.

Artigo submetido em 08.08.02, aceito em 13.02.03

\section{Abstract}

Objective: to evaluate the occurrence of osteopenia and the prognostic factors of bone mass in a pediatric group with type 1 diabetes.

Methods: the following parameters were analyzed in a group of 23 patients with type 1 diabetes aged $10.9 \pm 2.9$ years: bone mineral density, serum C peptide, glycosylated hemoglobin, serum calcium, serum alkaline phosphatase, serum phosphorus and calciuria. Clinical variables included age, weight, height, body mass index, pubertal stage, insulin doses, duration of diabetes and calcium intake. Bone mineral density was evaluated in the lumbar spine and the results were expressed in deviation standard score by age and sex. Calcium intake was calculated based on feeding report, body mass index was calculated using the "Quetelet" formula and pubertal stage was defined according to the Tanner-Whitehouse criteria. Simple linear regression was used to analyze correlations between variables and the Mann-Whitney U test was used to compare groups.

Results: average bone mineral density was normal $(-0.75 \pm 1.01$ SD). However we verified that $39.1 \%$ of the patients had osteopenia. When comparing data of osteopenic patients $(n=9)$ to non-osteopenic patients $(n=14)$, we observed that $C$ peptide of osteopenic group was higher than that of non-osteopenic group $(0.56 \pm 0.18$ versus $0.29 \pm$ $0.20 ; \mathrm{p}<0.05)$. Body mass index and $\mathrm{C}$ peptide correlated with bone mineral density. Duration of diabetes was inversely correlated with C peptide $(\mathrm{p}<0.01)$ and directly correlated with insulin doses $(\mathrm{p}<0.01)$.

Conclusion: osteopenia occurred in $39.1 \%$ of the patients with type 1 diabetes. The presence of osteopenia was related to higher levels of $\mathrm{C}$ peptide.

JPediatr(Rio J) 2003;79(3):253-8: type 1 diabetes, osteopenia, bone mineral density.

\section{Introdução \\ É durante a infância e a adolescência que se adquire grande parte do capital mineral do organismo; fases durante as quais observa-se $o$ aumento mais significativo da massa óssea. O aumento anual da densidade mineral óssea (massa óssea expressada em $\mathrm{g} / \mathrm{cm}^{2}$ ) é elevado durante os três primeiros anos de vida pós-natal, com diminuição progres-}


siva até o início da puberdade. Durante a puberdade, o aumento anual da densidade mineral óssea (DMO) eleva-se chegando a um máximo nos estádios III e IV de Tanner. Após a finalização da puberdade, o aumento anual da DMO diminui progressivamente, mantendo-se em níveis inferiores até a idade de 21-25 anos, quando a DMO estabiliza-se. Nesta fase, o indivíduo alcança seu pico de massa óssea. Do aumento total da densidade mineral óssea, $50 \%$ produz-se desde os primeiros meses da vida até o início do desenvolvimento puberal, $30 \%$ durante o desenvolvimento puberal e aproximadamente $20 \%$ durante a adolescência tardia, até a idade de 21-25 anos. A partir dos 21-25 anos, a DMO mantém-se constante até a $4^{\mathrm{a}}-5^{\mathrm{a}}$ década de vida, quando se inicia uma perda fisiológica gradual secundária ao avanço da idade que, nos indivíduos do sexo feminino, associa-se à perda peri e pós-menopausa ${ }^{1,2}$.

Osteopenia e osteoporose referem-se a dois estados patológicos caracterizados por diminuição da massa óssea. Segundo a Organização Mundial de Saúde, osteopenia caracteriza-se por uma densidade mineral óssea situada entre -1 e -2,5 desvios padrão da média alcançada nos adultos jovens. A osteoporose, por outro lado, define-se como uma DMO inferior a -2,5 desvios padrão da média, sendo que a presença de fraturas ósseas por fragilidade caracterizaria a osteoporose grave. Na faixa etária pediátrica, a redução da massa óssea é denominada osteopenia, que se refere a uma DMO situada abaixo de - 1 desvio padrão da média para a idade e sexo. $\mathrm{O}$ termo osteoporose não se aplica nesta faixa etária, uma vez que ainda não foi atingido o pico de massa óssea.

Considerando que $80 \%$ da massa óssea adquire-se durante a infância e a adolescência ${ }^{1,2}$, a idade pediátrica é um período crítico para a aquisição do conteúdo mineral do esqueleto. $\mathrm{O}$ aumento progressivo da massa óssea durante esta faixa etária decorre de um estado de anabolismo ósseo, resultado de um padrão metabólico ósseo e mineral característico desta idade. Este padrão pode alterar-se no curso de certas doenças, especialmente nas doenças crônicas, provocando redução da massa óssea já na faixa etária pediátrica. Várias doenças crônicas ${ }^{3-7}$ têm sido relacionadas a esta situação, entre elas o diabetes melito. A redução da massa óssea foi descrita tanto em portadores de diabetes melito tipo 1 (DM1) quanto em portadores de diabetes melito tipo 2 (DM2) $)^{8-11}$.

Os mecanismos fisiopatológicos relacionados à perda óssea no DM parecem incluir redução da atividade osteoblástica, alteração do metabolismo fosfocálcico, diminuição da síntese colágena ou produção reduzida de IGF-I e insulina ${ }^{12-16}$. Alguns estudos sugerem que pacientes com mau controle metabólico e longo tempo de evolução do $\mathrm{DM} 1^{17,18}$ apresentam maior risco de osteopenia. No entanto, outros autores não confirmam estes achados, não havendo consenso entre as variáveis relacionadas à perda óssea na população diabética.

Realizamos este estudo com o objetivo de verificar se a redução da massa óssea está presente no nosso grupo de crianças e adolescentes com DM1 e estudar a existência de fatores prognósticos da massa óssea.

\section{Pacientes e métodos}

Foram estudadas 23 crianças e adolescentes de um total de 32 pacientes com diagnóstico de DM1, segundo os critérios da Associação Americana de Diabetes, acompanhados no ambulatório de endocrinologia pediátrica da Universidade Regional de Blumenau. Nove pacientes recusaram-se a participar do estudo, e um paciente foi excluído, devido à presença de artefato no seu exame densitométrico. Dos 23 pacientes estudados, 9 eram do sexo feminino ( 8 púberes) e 14 do sexo masculino (quatro púberes). Após consentimento informado e aprovação pelo Comitê de Ética, os pacientes foram submetidos à avaliação da massa óssea, coleta de sangue para dosagem sérica de cálcio, fosfatase alcalina, fósforo, peptídeo C e hemoglobina glicosilada $(\mathrm{HbG})$, e coleta de urina para determinação de calciúria. As variáveis clínicas estudadas foram idade, peso, estatura, IMC, estágio puberal, ingestão diária de cálcio, duração do DM1 e dose diária de insulina. Considerou-se ingestão diária adequada de cálcio $800 \mathrm{mg} /$ dia.

Peso e estatura foram determinados utilizando-se balança antropométrica Filizzola ${ }^{\circledR}$ e expressos em desvio padrão da média para idade e sexo (DP), segundo as curvas de crescimento do NCHS. Os estágios puberais foram definidos segundo os critérios de Tanner-Whitehouse. O IMC foi calculado de acordo com a fórmula de "Quetelet": Peso $(\mathrm{kg}) /$ Estatura $^{2}$ (metros) e expressos em DP, segundo as curvas do NCHS. A dose de insulina diária foi dividida pelo peso corporal e expressa em UI/kg/dia, considerando dose total diária a soma da insulina NPH e insulina regular utilizada diariamente. Nos pacientes em que a dose de insulina regular variava de acordo com a glicemia capilar e/ou glicosúria, a dose foi calculada em função do número de unidades de insulina regular utilizada com maior freqüência. A ingestão diária de cálcio foi avaliada mediante a aplicação de recordatório alimentar de 24 horas.

A determinação do cálcio e fósforo séricos foi realizada pela técnica do eletrodo seletivo, a calciúria pela técnica colorimétrica, a fosfatase alcalina pela cinética, o peptídeo $\mathrm{C}$ pela quimioluminescência e a $\mathrm{HbG}$ pela Elisa por micropartículas. Os valores de referência para estas técnicas são apresentadas na Tabela 1 . A calciúria foi dividida pelo peso corporal e expressa em $\mathrm{mg} / \mathrm{kg} / \mathrm{dia}$.

A massa óssea de cada paciente foi avaliada através da densitometria óssea de radiografia de dupla energia, utilizando aparelho Lunar DPX-L. A massa óssea foi medida em coluna lombar entre $\mathrm{L}_{2}-\mathrm{L}_{4}$. A densidade mineral óssea (DMO), expressa $\mathrm{em} \mathrm{g} / \mathrm{cm}^{2}$, foi posteriormente transformada em desvio padrão da média para idade e o sexo, utilizando os valores de normalidade fornecidos pelo fabricante. Considerou-se osteopenia uma DMO inferior a -1DP.

Os pacientes foram posteriormente divididos em dois grupos de acordo com a presença ou não de osteopenia, a 
Tabela 1 - Valores de referência das determinações laboratoriais empregadas no estudo

\begin{tabular}{lll}
\hline Variável & Valor de referência & Unidade \\
\hline Cálcio sérico & $8,5-11,0$ & $\mathrm{mg} / \mathrm{dl}$ \\
Fósforo sérico & $2,0-6,1$ & $\mathrm{mg} / \mathrm{dl}$ \\
Fosfatase alcalina & $56-230$ & $\mathrm{U} / \mathrm{L}$ \\
HbG & $4,8-7,8$ & $\%$ \\
Peptídeo C & $0,9-4,0$ & $\mathrm{ng} / \mathrm{ml}$ \\
\hline
\end{tabular}

fim de se estudar possíveis diferenças entre as variáveis estudadas. O grupo de pacientes com osteopenia ficou constituído por 9 pacientes (quatro púberes, três do sexo feminino), e o grupo de pacientes sem osteopenia, por 14 pacientes ( 8 púberes, 6 do sexo feminino).

Os dados foram armazenados e analisados no programa estatístico Stat View II ${ }^{\circledR}$. Na caracterização da população, utilizou-se a estatística descritiva. No estudo das relações entre variáveis, foi utilizada a regressão linear simples. $\mathrm{Na}$ comparação entre grupos, foi utilizado o teste U de MannWhitney. Foi considerado nível de significação estatística os valores de "p" igual ou inferiores a 0,05 .

\section{Resultados}

As características clínicas dos 23 pacientes estudados são apresentadas na Tabela 2. Observou-se que a ingestão diária de cálcio foi inferior à recomendada em 16 pacientes.

Os níveis séricos de cálcio, fósforo e fosfatase alcalina foram normais, com médias de 9,4 $\pm 0,9 \mathrm{mg} / \mathrm{dl}, 4,3 \pm 0,9$ $\mathrm{mg} / \mathrm{dl}$ e $197,0 \pm 80,8 \mathrm{U} / \mathrm{L}$, respectivamente. A calciúria média foi de $2,8 \pm 3,1 \mathrm{mg} / \mathrm{kg} / \mathrm{dia}$, sendo a mediana 2,0 $\mathrm{mg} / \mathrm{kg} / \mathrm{dia}$. Um paciente do grupo não osteopênico apresentou calciúria elevada $(>4,0 \mathrm{mg} / \mathrm{kg} / \mathrm{dia})$. Dois pacientes do grupo osteopênico não coletaram urina para determinação da calciúria. A média do peptídeo $C$ foi de $0,40 \pm 0,2 \mathrm{ng} / \mathrm{ml}$.
Todos os pacientes apresentaram níveis de peptídeo $\mathrm{C}$ abaixo do valor de referência. A HbG teve média de 10,6 $2,9 \%$, com níveis séricos variando entre 6,3 a 17,3\%. Dois pacientes apresentaram valores normais de $\mathrm{HbG}$.

Observamos que a média da DMO foi normal $(-0,75 \pm$ 1,0 DP), variando de $-2,96$ a 1,08 DP. No entanto, verificamos que 9 pacientes $(39,1 \%)$ apresentaram osteopenia. Ao comparar os dados dos pacientes osteopênicos $(n=9)$ com os não osteopênicos ( $\mathrm{n}=14)$, observamos que o peptídeo C do grupo osteopênico foi superior ao do grupo não osteopênico (Figura 1). Não se evidenciaram diferenças significativas das demais variáveis estudadas entre os dois grupos (Tabela 3). Dos parâmetros clínicos e bioquímicos estudados, somente o IMC e o peptídeo C correlacionaram-se com a DMO (Figura 2). A duração do diabetes correlacionou-se negativamente com o peptídeo $\mathrm{C}(\mathrm{R}=-0,590 ; \mathrm{p}<0,01)$ e positivamente com a dose diária de insulina $(\mathrm{UI} / \mathrm{kg} / \mathrm{dia})(\mathrm{R}=$ $0,562 ; \mathrm{p}<0,01)$. Não houve diferença estatisticamente significativa da DMO dos pacientes púberes versus os não púberes.

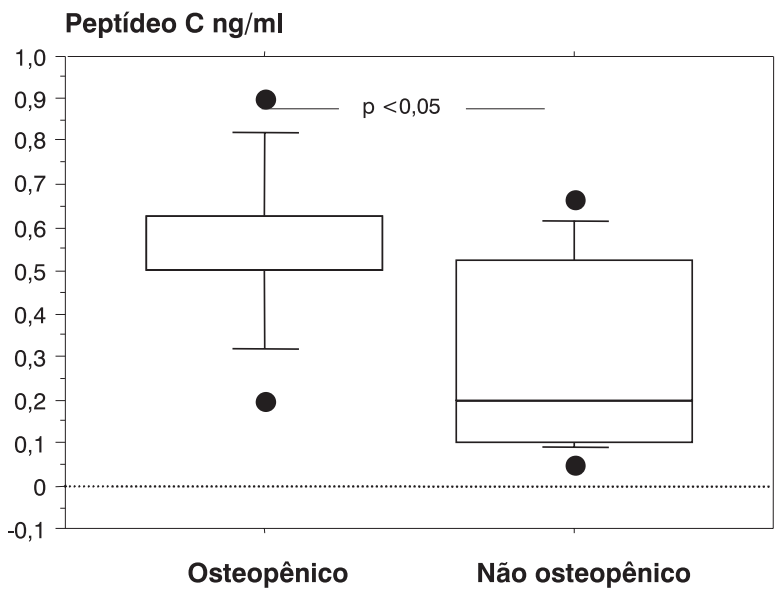

Figura 1 - Comparação dos níveis séricos de peptídeo C (média $\pm \mathrm{DP}$ ) nos dois grupos de pacientes

Tabela 2 - Características clínicas dos pacientes ( $\mathrm{n}=23)$

\begin{tabular}{lccc}
\hline Variável & Média & D.P.* & Intervalo \\
\hline Idade & 10,9 & 2,9 & $5,0-16,3$ \\
Duração do diabetes (meses) & 40,0 & 34,2 & $3-123$ \\
Dose de insulina (UI/kg/dia) & 0,92 & 0,26 & $0,30-1,29$ \\
Ingestão diária de cálcio (mg/dia) & 584,0 & 261,0 & $224-1074$ \\
Peso (DP) & $-0,47$ & 0,63 & $-1,29-0,70$ \\
Estatura (DP) & $-0,67$ & 0,53 & $-1,50-0,21$ \\
IMC (DP) & 0,03 & 0,86 & $-1,50-2,00$
\end{tabular}

* desvio padrão. 


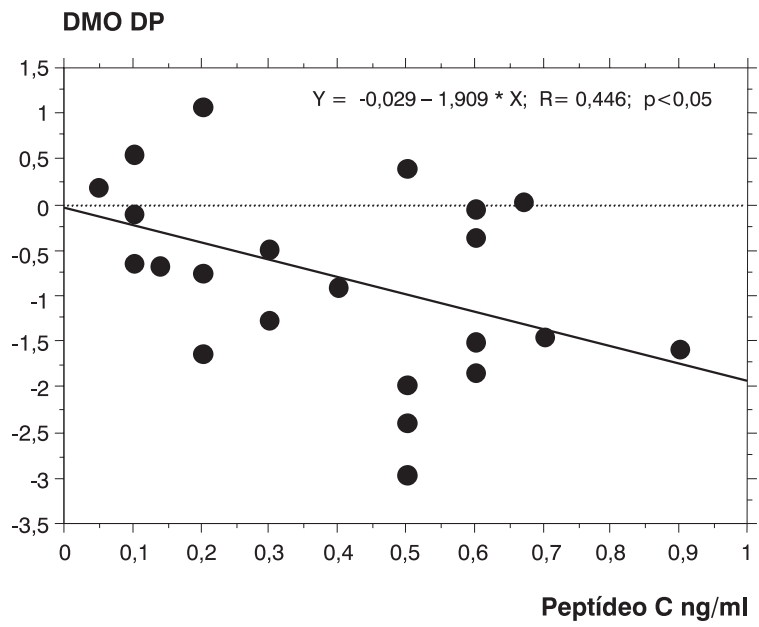

Figura 2 - Correlação entre DMO e peptídeo C

Embora se demonstre uma correlação significativa entre DMO versus IMC e peptídeo $C$, não se observa um valor de corte preciso de IMC ou peptídeo C, a partir do qual estivessem situados todos os pacientes osteopênicos.

\section{Discussão}

A perda da massa óssea tem sido reportada em pacientes com DM1 nas diferentes faixas etárias. Esta perda de massa óssea foi descrita tanto no tecido ósseo do tipo cortical como no tecido ósseo do tipo trabecular ${ }^{1921}$. Na idade pediátrica, encontram-se relatos de redução da massa óssea trabecular isoladamente ${ }^{8,22}$, ou associada à redução da massa óssea cortical ${ }^{8}$. Este estudo confirma os achados de redução da massa óssea trabecular em crianças e adolescentes portadores de DM1. No grupo estudado, 39\% dos pacientes apresentam osteopenia em coluna lombar. $\mathrm{Na}$ literatura revisada, esta cifra pode variar de 30 a $50 \%$ 8,9,23.

Estudos anteriores divergem com relação à associação entre o mau controle metabólico e a presença de osteopenia. Alguns estudos demonstram esta associação ${ }^{12,17}$, enquanto outros não ${ }^{8,24}$. Nós não encontramos correlação entre a DMO e os níveis séricos de HbG. É possível que o tamanho da amostra estudada tenha dificultado a obtenção de uma relação significativa entre tais variáveis, ou, neste grupo de pacientes, este fator não esteja envolvido na gênese da osteopenia.

A DMO não se correlacionou com a duração da doença. Os estudos em que esta relação é avaliada são discordantes. Parece, no entanto, que a perda de massa óssea relacionada ao diabetes ocorre em fases diferentes na evolução da doença, podendo ser de início recente ou tardio. A demonstração da perda de massa óssea em pacientes com DM1 de início recente nos sugere que exista um comprometimento do metabolismo ósseo decorrente da própria doença. Mac Nair e cols. ${ }^{12}$, estudando 215 diabéticos de todas as idades em uso de insulina, demonstraram que a perda óssea relacionada ao diabetes ocorre nos primeiros cinco anos de evolução. Há relato da presença de osteopenia em crianças com DM1 de duração inferior a dois anos ${ }^{8}$. Neste estudo, $90 \%$ dos pacientes osteopênicos tinham doença há menos de cinco anos, o que demonstra que no grupo estudado a perda óssea é um achado precoce. Recentemente, Gunczler e col. ${ }^{25}$ também demonstraram perda de massa óssea como um achado precoce no DM1.

O mecanismo exato responsável pela perda óssea em pacientes com diabetes não está totalmente elucidado. Alguns estudos demonstram que, durante a fase de crescimento, esta perda seja decorrente de um estado de baixo metabolismo ósseo ${ }^{8,15,26}$. Os marcadores do metabolismo

Tabela 3 - Comparação entre os dois grupos de pacientes

\begin{tabular}{lcc}
\hline Variável & $\begin{array}{c}\text { Não osteopênicos(n=14) } \\
\text { (média } \pm \text { DP) }\end{array}$ & $\begin{array}{c}\text { Osteopênicos (n=9) } \\
\text { (média } \pm \text { DP) }\end{array}$ \\
\hline Idade & $10,9 \pm 2,2$ & $10,3 \pm 3,8$ \\
Duração do diabetes (meses) & $50,0 \pm 7,1$ & $25,4 \pm 21,3$ \\
Dose de insulina (UI/kg/dia) & $0,94 \pm 0,22$ & $0,89 \pm 0,31$ \\
Ingestão diária de cálcio (mg/dia) & $564,2 \pm 244,7$ & $617,4 \pm 298,8$ \\
Estatura (DP) & $-0,75 \pm 0,47$ & $-0,87 \pm 0,53$ \\
IMC (DP) & $0,38 \pm 0,92$ & $-0,38 \pm 0,69$ \\
Peptídeo C (ng/ml) & $0,29 \pm 0,20$ & $0,56 \pm 0,18 *$ \\
HbG (\%) & $10,0 \pm 3,7$ & $11,4 \pm 2,9$ \\
\hline
\end{tabular}

${ }^{*} p<0,05$ 
ósseo encontravam-se dentro dos valores de normalidade, não havendo diferenças entre o grupo de pacientes osteopênicos e o de não osteopênicos. Alguns estudos sugerem que a osteopenia dos diabéticos de tipo 1 seja decorrente de baixos níveis de insulina ou de IGF-I ${ }^{12,15,27}$, ambos com ações anabólicas sobre o metabolismo ósseo, cuja redução seria compatível com o estado de baixo metabolismo ósseo descrito anteriormente. A presença de níveis superiores de peptídeo C, no grupo de pacientes com osteopenia, e a correlação inversa entre os níveis séricos de peptídeo $\mathrm{C}$ e a DMO poderiam sugerir que a insulinopenia esteja relacionada à perda óssea destes pacientes. Portanto, nas fases iniciais da doença, quando os níveis de peptídeo $\mathrm{C}$ são superiores, é o momento na evolução da doença que mais se aproxima da insulinopenia, que precede o diagnóstico do DM1. Isso nos leva a pensar que, nos pacientes estudados, este seja o fator determinante de sua perda óssea. Como comentado anteriormente, a osteopenia é um achado precoce no grupo de pacientes estudado, o que corrobora esta hipótese. MacNair e col. ${ }^{27}$, embora não tenham correlacionado peptídeo C com DMO, demonstraram um declínio progressivo da perda de massa óssea com os anos de evolução do DM1. Estes fatos não afastam a possibilidade de haver, no futuro, perda óssea relacionada ao mau controle metabólico, como descrito na literatura. É provável que a perda de massa óssea relacionada ao mau controle metabólico ocorra após vários anos de evolução, não atingindo a faixa etária pediátrica com frequiência. Os achados de aumento de DMO no hiperinsulinismo ${ }^{28,29} \mathrm{e}$ em pacientes diabéticos, com nefropatia terminal, quando a depuração de insulina diminui ${ }^{30}$, reforçam a idéia de que a insulinopenia esteja implicada na gênese da osteopenia no diabetes tipo 1 .

É provável que a perda de massa óssea decorrente do diabetes seja um processo multifatorial, como já sugerido por Martinez e cols. ${ }^{27}$ Portanto, vários fatores podem estar envolvidos, concomitante ou isoladamente, no aparecimento da osteopenia destes pacientes, podendo variar em função das características da população estudada. A baixa ingestão de cálcio encontrada nos pacientes estudados poderia ser um fator adicional na gênese da osteopenia, já que está bem estabelecido na literatura que uma ingestão adequada de cálcio é essencial para uma adequada aquisição mineral ${ }^{31}$. A observação de uma ingestão baixa de cálcio chama a atenção para uma inadequação alimentar, cuja correção eliminaria um fator de risco a mais para osteopenia no grupo estudado.

Em conclusão, os resultados deste estudo demonstram que a osteopenia está presente em crianças e adolescentes com DM1, ocorre nos primeiros anos de evolução da doença e está relacionada com níveis séricos superiores de peptídeo C. A presença de osteopenia, nesta faixa etária, pode prejudicar o pico de massa óssea dessas crianças; assim, seu seguimento a longo prazo nos permitirá saber se atingirão um pico de massa óssea adequado na idade adulta. A correção da ingestão diária de cálcio poderá eliminar um fator de risco adicional para perda de massa óssea neste grupo. Considerando que a amostra estudada é pequena, estudos futuros, incluindo maior número de pacientes, serão úteis para confirmação destes achados.

\section{Agradecimentos}

Os autores desejam expressar seus agradecimentos à Pró-Reitoria de Pesquisa e Pós-Graduação da FURB, especialmente ao Professor Ivo Theiss, Pró-Reitor de Pesquisa e Pós-Graduação durante o período de realização do estudo, e ao programa PIBIc-CNPq, ambos pelo apoio financeiro dispensado ao estudo.

\section{Referências bibliográficas}

1. del Rio L, Carrascosa A, Pons F, Gussinyé M, Yeste D, Domenech M. Bone mineral density of the lumbar spine in white Mediterranean Spanish children and adolescents: changes related to age, sex and puberty. Pediatr Res 1994;35:362-6.

2. Glastre C, Braillon P, David L, Cochat P, Meunier PJ, Delmas PD. Measurement of bone mineral content of the lumbar spine by dual energy X-ray absorptiometry in normal children: correlations with growth parameters. J Clin Endocrinol Metab 1990;70: 1330-3.

3. Audí L, Vargas DM, Gussinyé M, Yeste D, Martí G, Carrascosa A. Clinical and biochemical determinants of bone metabolism and bone mass in adolescents female patients with anorexia nervosa. Pediatr Res 2002;51:1-8.

4. Kaufman JM, Taelman P, Vermeulen A, Vandeweghe M. Bone mineral status in growth hormone-deficient male with isolated and multiple pituitary deficiencies of childhood onset. J Clin Endocrinol Metab 1992;74:118-23.

5. Fässler ALC, Bonjour JP. Osteoporosis as a pediatric problem. Pediatric Nutrition 1995;42:811-24.

6. Carrascosa A, Gussinyé M, Yeste D, del Rio L, Audí L, Enrubia $\mathrm{M}$, et al. Skeletal mineralization during infancy, childhood and adolescence in the normal population and in populations with nutritional and hormonal disorders. Dual X-ray absorptiometry (DEXA) evaluation. In: Schönau E, editor. Paediatric Osteology. New developments in diagnosis and therapy. Amsterdam: Elsevier Science; 1996.p.93-102.

7. van der Sluis IM, Keizer-Schrama SMPFM. Osteoporosis in childhood: bone density of children in health and disease. J Pediatr Endocrinol Metab 2001;14:814-59.

8. Gunczler P, Lanes R, Paz-Martinez V, Martinis R, Esaa S, Colmenares V, et al. Decreased lumbar spine bone mass and low bone turnover in children and adolescents with insulin-dependent diabetes mellitus followed longitudinally. J Pediatr Endocrinol Metab 1998;11:413-9.

9. Levin ME, Boissen VC, Aviolli LV. Effects of diabetes mellitus on bone mass in juvenile and adult onset diabetes. N Engl J Med 1976;294:241-5.

10. Buysschaert M, Cauwe F, Jamart J, Brichant C, Coster P, Magnan A, et al. Proximal femur density in type 1 and 2 diabetic patients. Diabete Metab 1992;18:32-7. 
11. Tuominen JT, Impivaara O, Puukka P, Rönnemaa T. Bone Mineral density in patients with type 1 and type 2 diabetes. Diabetes Care 1999;22:1196-200.

12. McNair P, Madsbad S, Christensen M, Christensen C, Faber O, Transbol, et al. Bone mineral loss in insulin treated diabetes mellitus: studies on pathogenesis. Acta Endocrinol 1979;90: 463-72.

13. Brenner RE, Riemenchneider B, Blum W, Möricke M, Teller WM, Pirsig W, et al. Defective stimulation of proliferation and collagen biosynthesis of human bone cells by serum from diabetic patients. Acta Endocrinol 1992;127:509-14.

14. Gregorio F, Cristallini S, Santeusanio F, Filippone P, Fumelli P. Osteopenia associated with non-insulin-dependent diabetes mellitus: what are the cause? Diabetes Res Clin Pract 1994;23: 43-54.

15. Bouillon R, Bex M, Van Herck E, Laureus J, Dooms L, Lesaffre $\mathrm{E}$, et al. Influence of age, sex and insulin on osteoblast function: osteoblast dysfunction in diabetes mellitus. J Clin Endocrinol Metab 1995;80:1194-202.

16. Glajchen N, Epstein S, Ismail F, Thomas S, Fallon M, Chakrabarti S. Bone mineral metabolism in experimental diabetes mellitus: osteocalcin as a measure of remodeling. Endocrinology 1988;123(1):290-5.

17. McNair P, Madsbad S, Christiansen C, Faber OK, Transbol I, Binder C. Osteopenia in insulin treated diabetes mellitus. Its relation to age at onset, sex and duration of disease. Diabetologia 1978;15:87-90

18. Mathiassen B, Nilsen S, Ditzel J, Rodbro P. Long-term bone loss in insulin-dependent diabetes mellitus. J Intern Med 1990; 227: 325-7.

19. Auwerx J, Dequeker J, Bouillon R, Geusens P, Nijs J. Mineral metabolism and bone mass at peripheral and axial skeleton in diabetes mellitus. Diabetes 1988;37:8-12.

20. Forst T, Pfüzner A, Kann P, Cchehler B, Lobmann R, Shäfer H, et al. Peripheral osteopenia in adults patients with insulindependent diabetes mellitus. Diabetic Medicine 1995;12:874-9.

21. Muñoz-Torres M, Jódar E, Escober-Jiménez F, López-Ibarra PJ, Luna JD. Bone mineral density measured by dual X-ray absorptiometry in Spanish patients with insulin-dependent diabetes mellitus. Calcif Tissue Int 1996;58:316-9.

22. Lettgen B, Hauffa B, Mohlmann C, Jeken C, Reiner C. Bone mineral density in children in adolescents with juvenile diabetes: using peripheral quantitative tomography. Horm Res 1995; 43:173-5.
23. Martinez DG, Pascual BJ, Hawkins CF. Estudio de la densidad mineral ósea en pacientes com diabetes mellitus tipo 1. Revista Hormona y Factores de Crescimiento 1995;1:32-6.

24. Pascual J, Argente J, Muñoz M. Bone mineral density in children and adolescents with diabetes mellitus type 1 of recent onset. Calcif Tissue Int 1998;63:31-5.

25. Gunczler P, Lanes R, Paoli M, Martinis R, Villaroel O, Weisinger JM. Decreased bone mineral density and bone formation markers shortly after diagnosis of clinical type 1 diabetes mellitus. J Pediatr Endocrinol Metabol 2001;14:525-8.

26. Krakauer JC, McKenna MJ, Buderer NF, Rao DS, Whitehouse FW, Parfitt AM. Bone loss and bone turnover in diabetes. Diabetes 1995;44:775-82.

27. McNair P, Christiansen MS, Christiansen S, Madsbad S, Faber $\mathrm{O}$, Binder $\mathrm{C}$, et al. Development of bone mineral loss in insulintreated diabetes: a $1 \frac{1 / 2}{2}$ years follow-up study in sixty patients. Eur J Clin Invest 1981;11:55-9.

28. Barrett-Connor E, Kritz-Silvertein. Does hyperinsulinemia preserve bone? Diabetes Care 1996;19:1388-92.

29. Stolk RP, van Daele PL, Pols HA, Burger H, Hofman A, Birkennhager JC, et al. Hyperinsulinemia and bone mineral density in an elderly population: The Rotterdam Study. Bone 1996;19:545-9.

30. Koshiyama H, Tanaka K, Nakao K. Is bone mineral density paradoxically increased in early stage of diabetic nephropathy. Arch Intern Med 1999;159:1145.

31. Matkovic V. Calcium and peak bone mass. J Intern Med 1992; 231:151-60.

Endereço para correspondência:

Dra. Deisi Maria Vargas

Rua Floriano Peixoto, 616 - Centro

CEP 89010-500 - Blumenau, SC

Tel/Fax: (47) 322.5533

E-mail: deisivargas@terra.com.br 